\title{
Developing Activities for Teaching Cloud Computing and Virtualization
}

\author{
Emre Erturk \\ Senior Lecturer, School of Computing \\ Eastern Institute of Technology \\ Napier, New Zealand \\ eerturk@eit.ac.nz
}

\author{
Bhuwan Maharjan \\ Postgraduate Student, School of Computing \\ Eastern Institute of Technology \\ Napier, New Zealand \\ bhuwankomail@gmail.com
}

\begin{abstract}
Cloud computing and virtualization are new but indispensable components of computer engineering and information systems curricula for universities and higher education institutions. Learning about these topics is important for students preparing to work in the IT industry. In many companies, information technology operates under tight financial constraints. Virtualization, (for example storage, desktop, and server virtualization), reduces overall IT costs through the consolidation of systems. It also results in reduced loads and energy savings in terms of the power and cooling infrastructure. Therefore it is important to investigate the practical aspects of this topic both for industry practice and for teaching purposes. This paper demonstrates some activities undertaken recently by students at the Eastern Institute of Technology New Zealand and concludes with general recommendations for IT educators, software developers, and other IT professionals.
\end{abstract}

Keywords-information technology; computer engineering; cloud computing; virtualization

\section{INTRODUCTION}

The recent 'cloud computing' paradigm incorporates some key aspects such as 'pay per use', high degree of availability, flexibility, scalability, and pervasiveness [1]. Cloud based resources also play an important role in online and mobile learning [2]. Cloud computing delivers a broad range of standardized and customizable applications and services via the internet. Infrastructure as a service (IaaS) is one of the basic models of service, and highly relevant to this paper. In IaaS, clients can outsource storage, hardware, servers and networking components from the provider. Aside from simplifying and outsourcing certain aspects of information technology (IT) operations and projects, cloud computing also inspires software developers to address the critical next-gen programming challenges of multi-cores, parallel programming and virtualization [1]. Conducting software testing within virtualized environments is becoming a more common practice. Cost saving is another attractive feature of developing software using resources and tools that are hosted on the cloud [3]. Traditional and in-house IT operations and projects required organizations to make upfront investments for infrastructure. This drawback can be by passed through present-day global and cloud based software development.
This paper's content is aimed at teaching cloud computing and virtualization by describing the experience from a course taught at the Eastern Institute of Technology New Zealand along with a brief theoretical background. Such a course should be considered as a broad course for students with a variety of interests and thus it should not formally require them to do any programming or coding scripts. The figures shown in this paper also provide a basic blueprint to the activities practiced during the course.

\section{UNDERSTANDING AND IMPLEMENTING VIRTUALIZATION}

A virtual machine is a representation of a real machine using software that provides an environment in which it can run. Through virtualization, server utilization is dramatically improved. Virtualization provides an opportunity for hardware resource sharing and multiple operating systems whereby generic hardware drivers are cloned and assigned to each virtual machine. A hypervisor is a software layer that logically partitions the physical hardware and guest operating systems. The latest trend of virtualization is the bare-metal approach where the hypervisor directly runs on the hardware without needing a host operating system $[4,5]$.

VMware and Xen are the most common virtualization products used by datacenters. These hypervisors offer a high level of security through a variety of features such as guest isolation, small code base, operating system separation, and privilege access. Furthermore, an important feature of the Xen hypervisor is its neutrality toward various operating systems and that it allows various operating systems (e.g. Linux, Solaris, BSD, etc.) to be the Domain0, which is used for configuring and controlling the virtualization activities [6].

Microsoft Azure (known as Windows Azure prior to being renamed) is a cloud computing platform and infrastructure for building, deploying and managing applications and services using Microsoft managed datacenters [7]. Virtual machines can be created using Microsoft Azure cloud infrastructure services. Subscribing to Azure is easy, robust, and suitable for a variety of users including students.

Figure 1 shows a logical pictorial design of a small datacenter where only few server racks are installed. It is divided into three layers. The top layer is a consumer network 
where all of the virtual PCs are connected to a local area network (LAN). Below this layer, there is a VMware vSphere Distribution Switch (VDS). The VDS abstracts the physical network and provides access-level switching in the vSphere hypervisor. This is useful for network virtualization because it enables logical networks that are independent of physical constructs [8]. Finally, Figure 1 illustrates the creation of a Virtual Local Area Network (VLAN), where the physical network is logically divided into two different VLANs (Bhuwan-VLAN-1 and Bhuwan-VLAN-2). These VLANs work above the routing and switching layer (i.e. Layer 3). Layer 2 devices are mainly smart switches that are used to interconnect the datacenter's Layer 3 components with the core layer (i.e. Layer 1) switches.

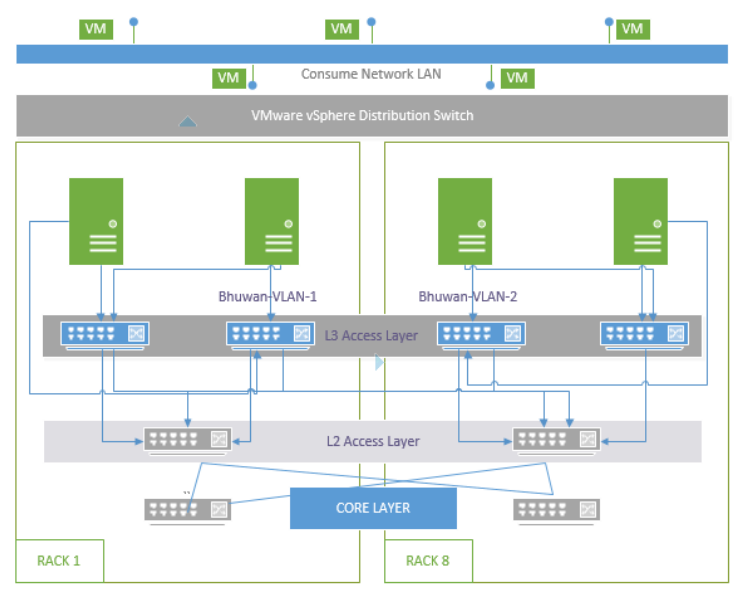

Fig. 1. Designing a small data center.

\section{LEARNING ACTIVITIES USING MICROSFT AZURE}

Figure 2 shows a Windows Server 2012 machine with eight processor cores and 14 GB of RAM. The domain name server (DNS) address for this virtual machine was set as bhuwan.cloudapp.net. The next task (shown in Figure 3) is to name and configure this virtual server. The virtual machine in this example is called sqlserver. Then PowerShell (for managing operating system and administrative tasks) and Remote Desktop Services for the virtual machine are configured as shown in Figure 4. This happens prior to provisioning and running the new virtual server. There is also an option to choose and add security extensions to further enhance and protect the virtual machine.

Students (or trainees) can also create a virtual network to connect many virtual machines and databases. In this network example, four different sub-networks are created as shown in Figure 5. Each subnet may have a fixed number of usable Internet Protocol (IP) addresses. For example, in the BhuvSubnet-4, the Classless Inter-Domain Routing (CIDR) value is set to /29. This provides just eight IP addresses to be managed by this new host. The last column in Figure 5 shows the actual IP addresses that are available.

After the creation of virtual resources, it is important for students to work on setting up user permissions and access.
This is called an Active Directory (AD). Here the existing DNS name i.e. bhuvmhzoutlook.onmicrosoft.com can be used for the AD domain name. New users can be added and be assigned with different roles. Figure 6 shows how a new user profile created and given a user administrator role. Figure 7 shows how a remote connection can be made to the virtual machine called bhuwanji. After logging onto the bare-metal virtual machine, the information observed are shown in Figure 8. Finally, Figure 9 shows an example of how students can use the Power Shell Integrated Scripting Environment (ISE) to interact with the server using a command line interface.

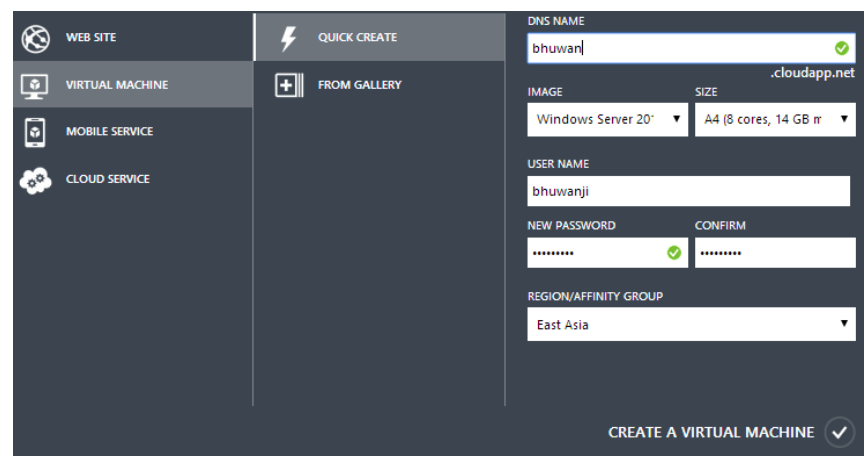

Fig. 2. Creating a new virtual machine.

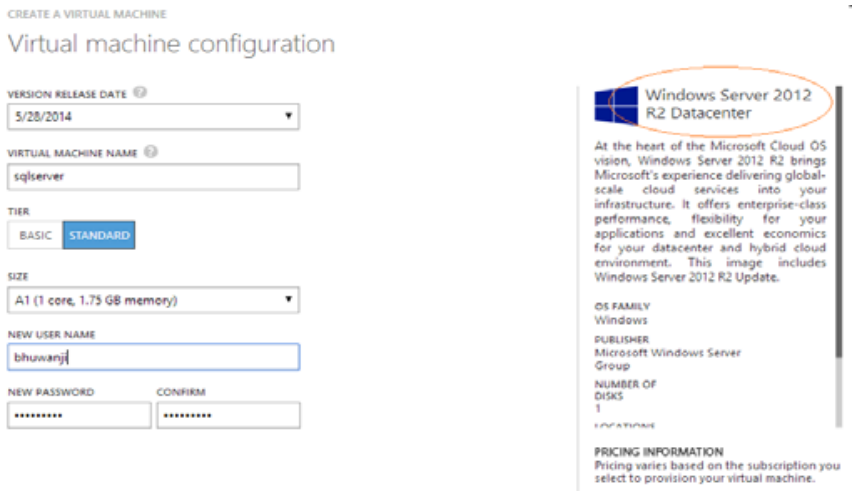

Fig. 3. Configuring a virtual machine.

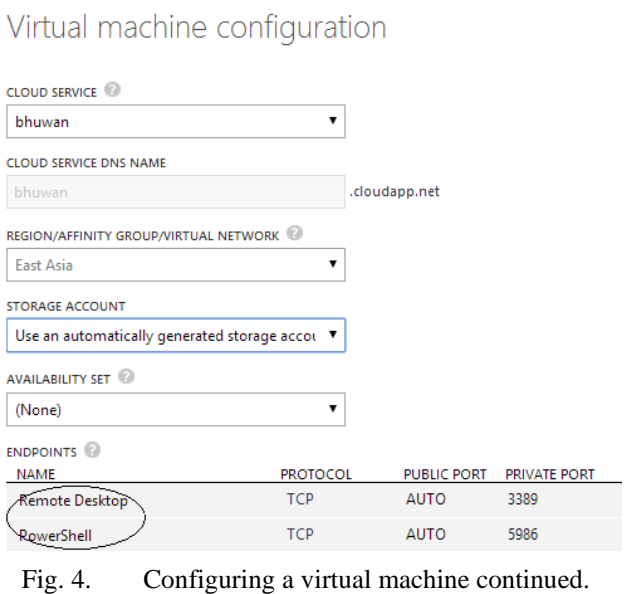




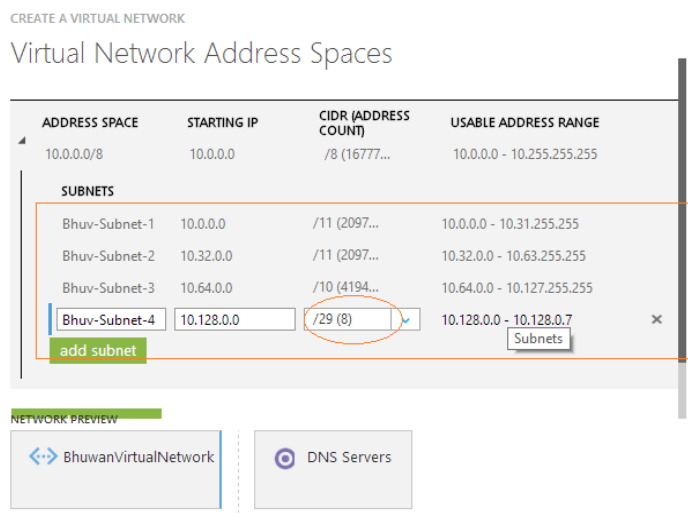

Fig. 5. Creating a virtual network.

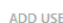

user profile

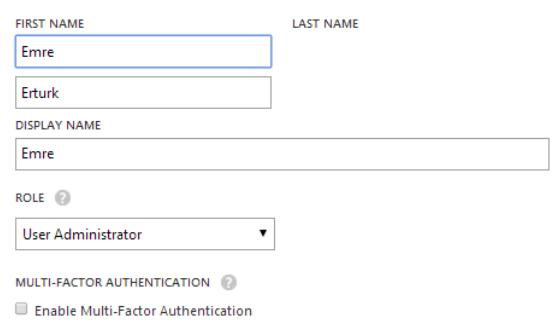

Fig. 6. Creating new users.

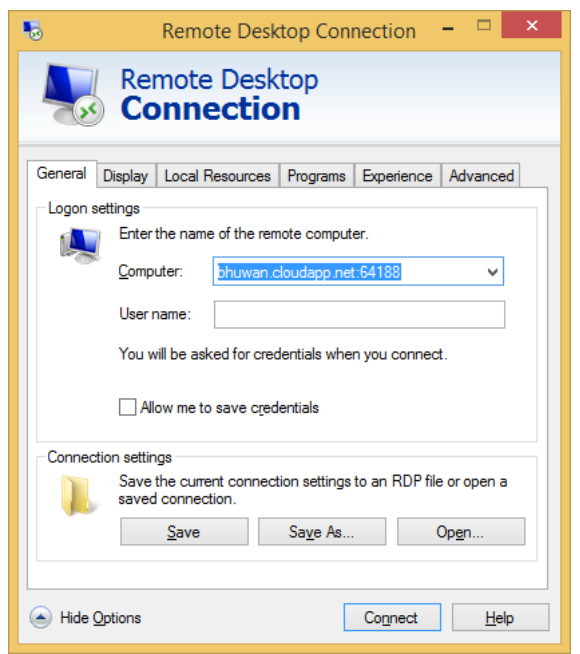

Fig. 7. Making a remote desktop connection.

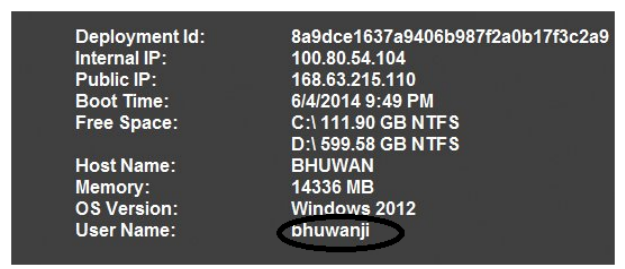

Fig. 8. Virtual system information.

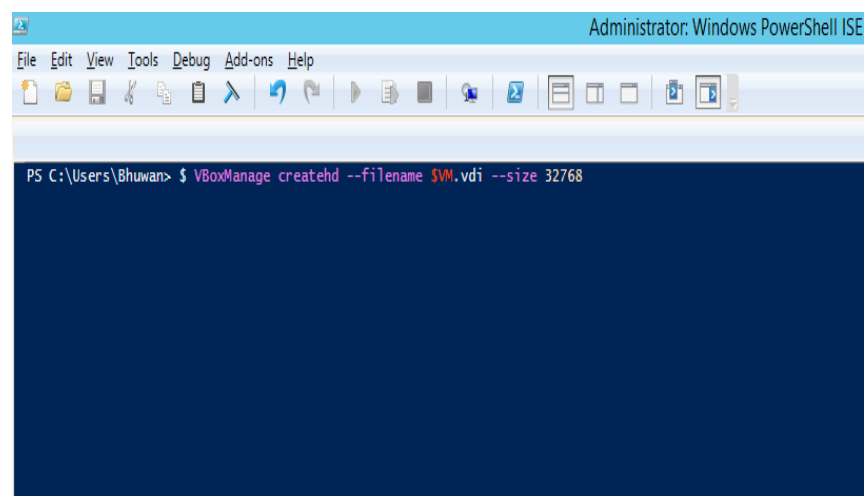

Fig. 9. Virtual system administration.

\section{CONCLUSION}

The practical work that is involved in producing the types of results shown in this paper requires initial familiarization, trouble-shooting, and self-guided technical learning for students and providing solid background beforehand should be considered necessary. One tutor should be sufficient for a number of ten students. Learning also involves students understanding the bigger picture, and teachers need to appeal to students' overall business perspectives and values. It needs to be emphasized that cloud computing and virtualization offer additional benefits in terms of freeing up physical resources, migrating resources and services elsewhere, and enhancing disaster recovery. These tools and ideas also have positive implications for the environment, which many students tend to appreciate. Each company is a unique case; if virtualization is considered and implemented appropriately for a given scenario, energy consumption for power and cooling can be better controlled. This paper also recommends software developers to consider using online resources and virtual environments for building and testing. This is more relevant in the current market with ever increasing numbers of web based applications, mobile applications, and software-as-a-service (SaaS) provisions. In conclusion, cloud computing has moved from being an initial hype to providing greater productivity to companies. Overall, the cloud continues to offer new opportunities to a diverse group of IT professionals.

\section{REFERENCES}

[1] P. Yara, R. Ramachandran, G. Balasubramanian, K. Muthuswamy, D. Chandrasekar, "Global Software Development with Cloud Platforms", Software Engineering Approaches for Offshore and Outsourced Development, Lecture Notes in Business Information Processing, Vol. 35, pp. 81-95, 2009

[2] E. Erturk, "An Intelligent and Object-oriented Blueprint for a Mobile Learning Institute Information System”, International Journal for Infonomics, Vol. 6, No. 3-4, 2013

[3] N. Oza, J. Münch, J. Garbajosa, A. Yague, E. Ortega, “Identifying Potential Risks and Benefits of Using Cloud in Distributed Software Development”, Product-Focused Software Process Improvement, Lecture Notes in Computer Science, Vol. 7983, pp. 229-239, 2013

[4] D. Bouley, "Impact Of Virtualization On Data Center Physical Infrastructure”, The Green Grid Library \& Tools, White Paper \#27, pp. 1-10, 2010 
[5] Microsoft Corporation, "Datacenter Virtualization", http://download.microsoft.com/download/a/7/a/a7ad1735-40d7-45089462-bcb79577b17b/virtualization_whitepaper_v2_1.pdf

[6] S. Spector, "Why Xen?: Xen Compared to Other Hypervisors", http://www-archive.xenproject.org/files/Marketing/WhyXen.pdf

[7] M. Tulloch, Introducing Windows Azure for IT Professionals, Microsoft Press, 2013

[8] VMware, "VMware Network Virtualization Design Guide", http://www.vmware.com/files/pdf/techpaper/Virtual-Network-DesignGuide.pdf

\section{AUTHORS PROFILE}

Emre Erturk received his Ph.D. from the University of Oklahoma in 2007. He has later taught as an Assistant Professor with the University of Maryland (USA) in the areas of business, economics, and IT. Currently, he is a Senior IT Lecturer at the Eastern Institute of Technology, New Zealand.

Bhuwan Maharjan is a postgraduate student at the Eastern Insittute of Technology in New Zealand. He is also an avid IT professional and software developer. He has previously worked at the College of Information Technology \& Engineering in Kathmandu, Nepal. 